\title{
Ultrasonography for the Diagnosis of Carpal Tunnel Syndrome in Diabetic Patients: Missing the Mark?
}

\section{Ultraschall zur Diagnose des Karpaltunnelsyndroms bei Patienten mit Diabetes: Treffen wir ins Ziel?}

Authors

Fabian Steinkohl', Alexander Loizides ${ }^{1}$, Leonhard Gruber ${ }^{1}$, Michael Karpf ${ }^{1}$, Gabriele Mörsdorf², Ingrid Gruber², Bernhard Glodny ${ }^{1}$, Wolfgang Löscher ${ }^{3}$, Hannes Gruber ${ }^{1}$

Affiliations

1 Department of Radiology, Medical University Innsbruck, Austria

2 Department of Vascular Surgery, Medical University Innsbruck, Austria

3 Department of Neurology, Medical University Innsbruck, Austria

Key words

Carpal Tunnel Syndrome, Diabetes Mellitus, ultrasound, Wrist-to-Forearm-Ratio, median nerve

received 20.08.2017

accepted 22.05.2018

Bibliography

DOI https://doi.org/10.1055/a-0639-5713

Published online: 2.7.2018

Fortschr Röntgenstr 2019; 191: 117-121

(c) Georg Thieme Verlag KG, Stuttgart · New York

ISSN 1438-9029

Correspondence

Dr. Alexander Loizides

Department of Radiology, Innsbruck Medical University,

Anichstrasse 35, 6020 Innsbruck, Austria

Tel.: ++ 43/5 12/504/22761

Fax: ++ 43/512/504/227 58

alexander.loizides@i-med.ac.at

\section{ZUSAMMENFASSUNG}

Ziel Diabetes mellitus (DM) und das Karpaltunnelsyndrom (CTS) sind häufige Erkrankungen. Die sonografische Diagnose des CTS basiert auf der Wrist-to-Forearm-Ratio (WFR). Kann man aber die WFR bei Patienten mit DM anwenden?

Material und Methoden Es wurden 233 Handgelenke von 153 Patienten untersucht. Die Querschnittsfläche (CSA) des Nervus medianus wurde mit einem Linearschallkopf erhoben. Die WFR wurde errechnet.

Ergebnisse Patienten mit DM und CTS hatten signifikant niedrigere WFR-Werte als Patienten ohne DM mit CTS $(p=0,002)$. Es gab keinen Unterschied zwischen der WFR von Patienten mit DM und ohne CTS und von Patienten mit DM und mit CTS $(p=0,06)$. Die diagnostische Genauigkeit zwischen Patienten mit DM und mit CTS und Patienten mit DM und ohne CTS war mit der WFR niedrig (ROC AUC = 0,630, $95 \% \mathrm{Cl} 0,541-0,715$, $p=0,011$ ).

Schlussfolgerung Die Ergebnisse dieser Arbeit legen nahe, dass die WFR bei Patienten mit DM und CTS eine niedrige diagnostische Genauigkeit hat. Daher sollte sie bei diesen Patienten nur mit Vorsicht verwendet werden.

\section{Kernaussagen}

- Die diagnostische Genauigkeit der WFR ist bei Diabetikern niedrig

- Die WFR sollte bei Patienten mit DM nicht verwendet werden

- Die sonografische Beurteilung des Nervus medianus sollte bei Patienten mit DM den Schwerpunkt auf morphologische Veränderungen legen

\section{ABSTRACT}

Purpose Diabetes mellitus (DM) and carpal tunnel syndrome (CTS) are common pathologies. The diagnosis of CTS can be facilitated by the use of an ultrasound-based wrist-to-forearm ratio (WFR) of the nerve diameter. However, the applicability of WFR in DM-patients is not yet clear.

Materials and Methods 233 wrists of 153 patients were examined. Cross-sectional areas (CSA) of the median nerve were obtained using a linear array probe. The WFR was calculated. Results Diabetics with CTS had significantly lower WFR values than non-diabetics with CTS $(p=0.002)$. There was no difference between the WFR of diabetics with and without CTS $(p=0.06)$. The diagnostic accuracy between diabetics with and without CTS was low for measurements of WFR (ROC AUC $=0.630,95 \% \mathrm{Cl} 0.541-0.715, \mathrm{p}=0.011$ ).

Conclusion Our findings suggest that the WFR has a low diagnostic accuracy in diabetic patients with CTS and should be used with caution in those patients.

\section{Key Points}

- The diagnostic accuracy of WFR is low in patients with DM

- WFR should not be used in patients with DM

- The sonographic evaluation of the median nerve in patients with DM should focus on morphological changes 


\section{Citation Format}

- Steinkohl F, Loizides A, Gruber L et al. Ultrasonography for the Diagnosis of Carpal Tunnel Syndrome in Diabetic Patients:

Missing the Mark?. Fortschr Röntgenstr 2019; 191: 117-121

\section{Introduction}

Carpal tunnel syndrome (CTS) is the most frequent nerve entrapment syndrome and accounts for over $90 \%$ of all entrapment neuropathies [ 1 - 3]. It has a prevalence of $14.4 \%$ [4] and is diagnosed predominantly clinically. The diagnosis is supported by nerve conduction studies (NCS) and ultrasound (US) whereupon some authors even propose the use of US as an alternative to NCS [5]. There are different approaches to diagnose a CTS using ultrasound, most of which rely on an increased cross-sectional area of the median nerve (CSA) at the level of the carpal tunnel (CT) [6-8]. A range of cut-off values to clearly identify patients with CTS have been proposed, but they vary considerably among different studies [9, 10]. In 2008 Hobson-Webb introduced a ratio calculated by the CSA at the level of CT and the CSA $12 \mathrm{~cm}$ proximally in the forearm. Those two measurements are used to calculate the so-called wrist-to-forearm ratio (WFR). A WFR greater than 1.4 is considered abnormal, i. e., diagnostic for CTS [11].

Diabetes mellitus (DM) has a worldwide prevalence of $8.7 \%$ [12] and is known to cause peripheral polyneuropathies (PNP): in fact, peripheral nerve involvement occurs in $50 \%$ of patients suffering from DM [13]. There is an ongoing debate whether DM causes sonographically visible changes in peripheral nerves. Experimental studies in rats showed no clear changes in the microscopic structure of nerves [14]. A more recent study did not show significant differences in the size or echogenicity of the fibular and tibial nerves in patients with diabetic PNP [15] while another study presented contradictory results such as an increase in the CSA of median nerves in patients with diabetic PNP in comparison with healthy controls [16]. Dyke et al. described morphometric abnormalities in the microvessels of nerves with relation to DM [17]. A recent study has shown that nerves are on average slightly enlarged compared to healthy subjects in patients with DM [18]. Nerve enlargement has been found in other neuropathies such as hereditary neuropathies like POEMS or metachromatic leukodystrophy or acquired conditions such as leprosy or sarcoidosis [19]. WFR is, among others, a widely used method to sonographically support the diagnosis of CTS or even exclude the necessity for surgical repair and thus triages patients. It is presently not known whether WFR delivers reliable results in diabetic patients.

It is unclear whether DM has an effect on nerve size or not. Watanabe et al. compared the CSA of peripheral nerves in diabetic patients with PNP and healthy volunteers. They describe an increase of the CSA in diabetic patients [16]. Based on this assumption, this paper aimed at evaluating the role of ultrasound in diabetic patients with CTS using larger cohorts than Watanabe et al.

The aim of this study was to elucidate whether the calculation of the WFR is suitable also in diabetic patients.

\section{Materials and Methods}

In this retrospective study based on our established standardized US assessment daily routine algorithm, we analyzed 277 wrists in 156 patients (average age: $64.3 \pm 13.8$ years, range: 25 to 93 years). Among those, 92 were female and 64 male. Patients had been examined between March 2010 and December 2015. Institutional review board approval was granted by means of a general waiver for studies with retrospective data analysis (Ethikkommission, Med. Univ. Innsbruck; 2009/02/20). Three groups of patients were included: Patients with clinically and electrophysiologically diagnosed CTS and no history of DM (Group DM-CTS+, 30 wrists), patients with DM and no history of CTS (Group DM+CTS-, 40 wrists), and patients with DM and CTS (Group DM+CTS+ 207 wrists). Patients were classified as suffering from DM II when insulin-dependent type II diabetes (according to clinical records) and a history of elevated HbA1c levels were evident.

All ultrasound assessments had been performed in a standardized fashion with a Philips iU22 or a Philips Epiq 7 machine (Philips, Bothell, Washington, USA) using a $12-5 \mathrm{MHz}, 17-5 \mathrm{MHz}$ or 18 $5 \mathrm{MHz}$ broadband linear array probe under standard presets dependent on availability and imaging requirements. The patients sat opposite the examiner, and the relevant arm was conveniently flexed with the wrist resting in a supinated position on a cushion so that the palmar part of the wrist and the forearm were accessible. CSA measurements of the median nerve were obtained in a standardized fashion at the median nerve's maximum swelling point, which was located at the proximal border of the flexor retinaculum. In order to calculate the WFR, the CSA was determined at the forearm $12 \mathrm{~cm}$ proximal to the level of the pronator quadratus muscle (PQ) as proposed by Hobson-Webb [11]. CSAs were measured by free-hand tracing of the median nerve including the hyperechoic epineurium using the on-board measurement tools. The wrist-to-forearm ratio was then calculated.

All images were stored in the institution's PACS (Agfa Impax, Agfa Healthcare, Mortsel, Belgium). Information on patient age, sex, most recent HbA1c in \% (available in 133 patients) and clinical diagnosis of PNP was extracted from the hospital information system.

\section{Statistical Analysis}

Statistical analysis was performed in GraphPad Prism Pro 6.05 (GraphPad Software Inc.; La Jolla, CA, USA) and SPSS version 22.0 (IBM Corp., Armonk, NY, USA). P-values < 0.05 were considered significant and $95 \%$ confidence intervals are given where appropriate.

Descriptive analysis was performed on all patients and subgroups. Group differences were calculated by a chi-square test in case of categorical variables, and by a two-sided t-test or a one- 
- Table 1 Group characteristics in regard to age, sex, presence of polyneuropathy and HbA1c levels for Group DM+CTS-, Group DM-CTS+, and Group DM+CTS+.

- Tab. 1 Charakteristika bezüglich Alter, Geschlecht, Vorliegen einer Polyneuropathy und HbA1c-Levels für die Gruppen DM+CTS-, Group DM-CTS

+ , und Group DM+CTS+.

\begin{tabular}{|c|c|c|c|c|}
\hline & group DM+CTS- & group DM-CTS+ & group DM+CTS+ & p-value \\
\hline age [years] & $68.2 \pm 10.3$ & $67.4 \pm 16.5$ & $63.8 \pm 13.6$ & $0.09^{1}$ (n. s.) \\
\hline sex (male) [\%] & 47.4 & 75.0 & 41.0 & $0.017^{2}$ \\
\hline clinical diagnosis of PNP [\%] & 15.4 & 10.5 & 12.7 & $0.001^{2}$ \\
\hline HbA1c [\%] & $7.76 \pm 1.64$ & - & $7.29 \pm 1.41$ & $0.07^{3}$ (n.s.) \\
\hline
\end{tabular}

way ANOVA with a Bonferroni-correction for multiple testing in case of continuous variables.

Box plots were generated to visualize the distribution of WFR and CSAs at the CT and at the PQ among asymptomatic diabetics, non-diabetic patients and diabetic patients with CTS. To compare the distribution of median nerve CSAs at the CT and PQ and WFR were analyzed on a wrist basis with a one-way ANOVA with a Bonferroni-correction for multiple comparisons after log2-transformation of the data set to achieve a Gaussian distribution (D'Agostino-Pearson omnibus-test for normality $>0.05$ for each data set).

Additionally, the correlation between HbA1c levels and WFR/ median nerve CSAs at the CT and PQ was visualized through scatter plots. A linear regression analysis was performed to detect correlations. Results are given as slope and $R^{2}$.

Finally receiver-operating characteristics (ROC) curves were used to assess the diagnostic accuracy of WFR and CSA measurements in the identification of patients with CTS. The results are given as area under the curve (AUC), sensitivity, specificity and likelihood ratio (LR). Ideal cut-offs were determined by means of Youden indices.

\section{Results}

\section{Cohort characteristics}

As described above, the patient wrists were grouped in three categories: Wrists from patients with clinically and electrophysiologically diagnosed CTS (Group DM-CTS+), patients with DM (Group DM+CTS-) and patients with DM and CTS (Group DM+ (TS+). Details on group characteristics are provided in $>$ Table 1.

There was no significant difference in regard to age between the three groups. There were significantly more male patients in Group DM. In Group DM more patients were diagnosed with PNP than in the other groups.

\section{Median nerve measurements}

There were no significant differences between median nerve CSAs of Group DM-CTS+ $\left(20.3 \pm 7.4 \mathrm{~mm}^{2}\right)$ and Group DM+CTS- (18.8 $\left.\pm 5.0 \mathrm{~mm}^{2}, p>0.999\right)$ and Group DM+CTS $+\left(18.3 \pm 7.4 \mathrm{~mm}^{2}\right.$, $\mathrm{p}=0.354)$. The difference between CT CSA did not differ significantly between Group DM+CTS+ and Group DM+CTS- ( $>0.999)$.

Furthermore, Group DM+CTS- had a significantly higher median nerve CSA at the PQ $\left(10.6 \pm 1.9 \mathrm{~mm}^{2}\right)$ than Group CTS $(8.8$ $\left.\pm 2.6 \mathrm{~mm}^{2}, \mathrm{p}=0.003\right)$ and Group DM+CTS+ $\left(9.3 \pm 3.0 \mathrm{~mm}^{2}\right.$, $\mathrm{p}=0.013$.

Both Group DM+CTS- $(1.79 \pm 0.48)$ and Group DM+CTS+ $(2.04 \pm 0.66, p=0.002)$ had significantly lower WFR values than Group DM-CTS+ $(2.37 \pm 0.75, p=0.023)$, yet there was no significant difference between the WFR values of Group DM+CTS- and Group DM+CTS+ $(p=0.154)(\triangleright$ Fig. 1).

Grouped by $\mathrm{HbA} 1 \mathrm{c}$ levels, no significant difference in regard to WFR, CSA at the CT or at the PQ could be observed except for significantly higher values for median nerve CSA at the PQ $(p=0.034)$ and significantly lower values for WFR $(p=0.008)$ in Group DM+CTS- compared to Group DM-CTS+. Linear regression showed no correlation between HbA1c levels and WFR $(0.028$ $\left.\pm 0.031, p=0.84, R^{2}=0.0001\right)$, median nerve CSAs at the CT $\left(0.557 \pm 0.287, P=0.06, R^{2}=0.0107\right)$ or $P Q(0.098 \pm 0.128$, $\left.p=0.443, R^{2}=0.0040\right)$.

\section{Diagnostic accuracy of median nerve measurements}

Diagnostic power to discriminate between Group DM+CTS+ and Group DM+CTS- was low both for measurements of WFR (ROC AUC $=0.630,95 \% \mathrm{Cl} 0.541-0.715, \mathrm{p}=0.011)$ and median nerve CSA at the CT (ROC AUC $=0.541,95 \% \mathrm{Cl} 0.455-0.627$, $\mathrm{p}=0.413$ ) (see $>$ Fig. 2). At a WFR cut-off of 1.4 (as suggested by the literature), the sensitivity was $84.5 \%$ (95 Cl 78.8-89.1\%), the specificity was $20.0 \%$ (95\% Cl $9.1-35.7 \%)$ and the likelihood ratio was 1.056. At a cut-off value of $16 \mathrm{~mm}^{2}$ median nerve CSA at the carpal tunnel (determined by the highest Youden index), the sensitivity was $70.0 \%$ (95 Cl $53.5-83.4 \%$ ), the specificity was $43.2 \%(95 \% \mathrm{Cl} 36.3-50.3 \%)$ and the likelihood ratio was 1.232 . 

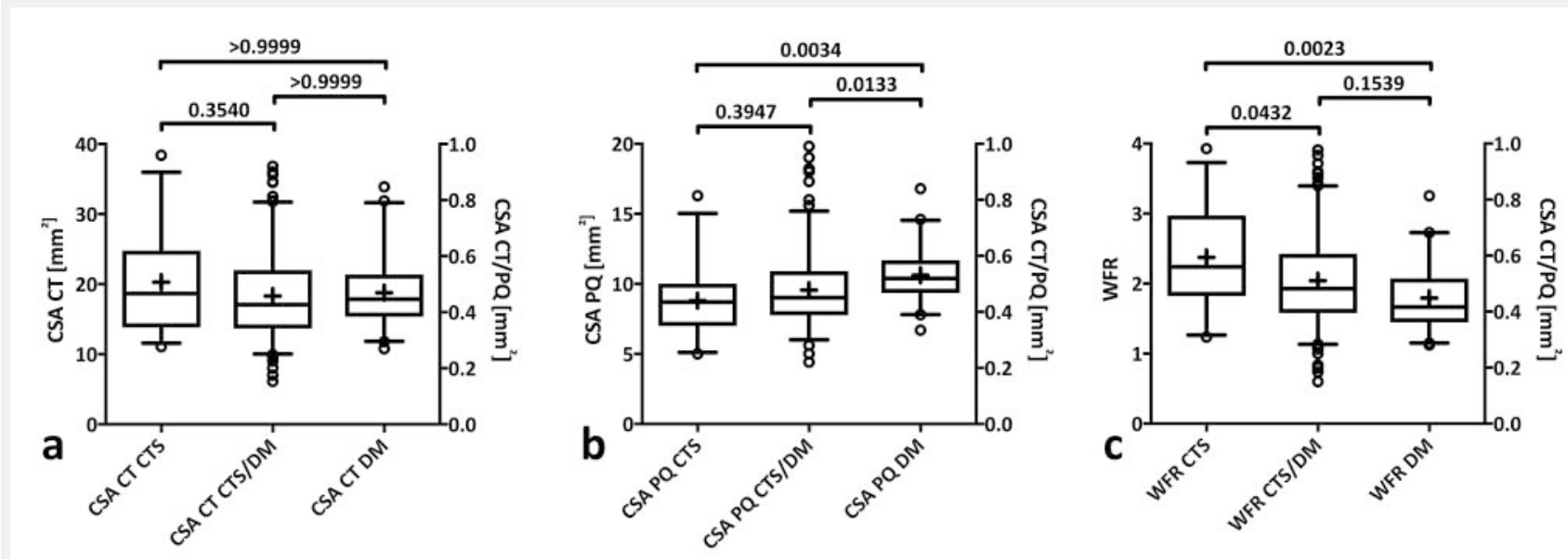

- Fig. 1 Differences in CSA at CT a; in CSA at PQ b; in WFR $\mathbf{c}$ in patients with CTS, in patients with CTS and DM and in patients with DM.

- Abb. 1 Unterschiede zwischen CSA im CT a; CSA auf Höhe des PQ b; und zwischen der WFR c in Patienten mit CTS, Patienten mit CTS und DM und Patienten mit DM.
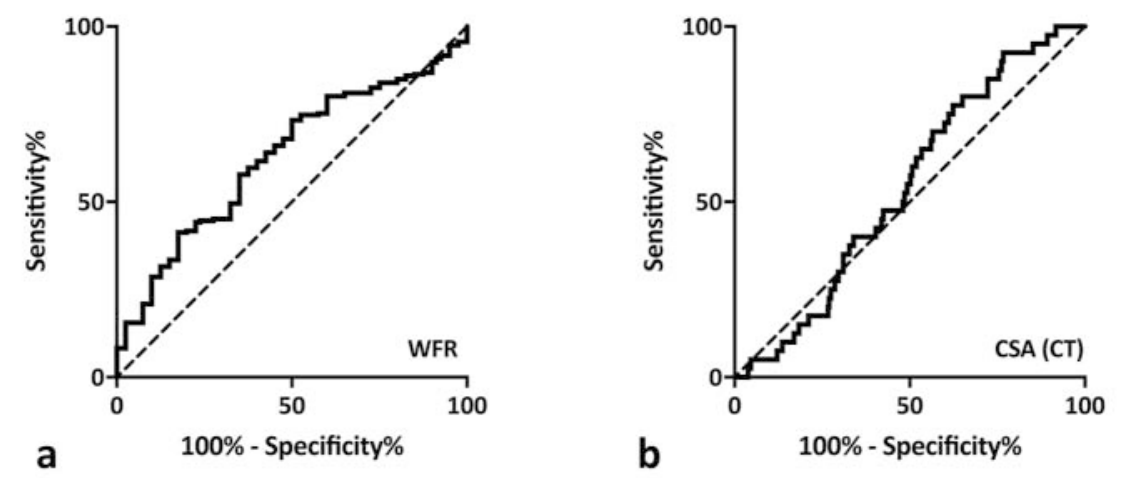

- Fig. 2 ROC curves for the correct identification of CTS in diabetics by WFR a or median nerve CSA at the carpal tunnel (CT) b.

- Abb. 2 ROC-Kurven für die richtige Erkennung von CTS in Patienten mit DM mit Hilfe der WFR a oder der CSA des Nervus medianus im Karpaltunnel (CT) b.

\section{Discussion}

We found that patients with DM had a larger proximal CSA and a lower WFR than patients without DM. However, due to the size of our cohorts, the data is not sufficient to confirm Watanabe's findings regarding enlargement of the median nerve in diabetic patients. Probably, further studies with even more participants on this topic are needed.

We used HbA1c levels as a marker for severity of DM. We were unable to detect a significant correlation between HbA1c levels and nerve CSA, even though there was a trend towards a greater CSA at the PQ in diabetic patients. This may be due to several reasons: our samples were very heterogeneous, we did not record duration of DM in diabetic patients, and we did not have sufficient records of older HbA1c levels from our patients.

As described above, the WFR is a ratio of the CSA measured at the CT and at the forearm, i. e., the nerve is its own control regard- ing a possible enlargement of CSA at CT. As we know, this enlargement occurs in non-diabetic patients with CTS [20-22]. Our data shows no significant difference in WFR between patients with DM and without CTS and patients with DM and CTS. Therefore it is impossible to diagnose CTS by means of US in diabetic patients. This finding is underlined by the ROC curves, which show that the chance to diagnose CTS in diabetic patients using WFR is like "flipping a coin".

We postulate the following mechanism to be responsible for these findings: The nerve is diffusely enlarged in the forearm in diabetic patients as found by Watanabe and colleagues [16]. Therefore, in diabetic patients the CSA at the forearm is preliminarily larger than in healthy controls. Enlargement of the median nerve in the CT, the site of entrapment in the case of CTS, is probably limited (e.g. by the epineurium or surrounding anatomic structures such as the subcutaneous fascia or even the transverse 
carpal ligament?). Although the difference between the CSA at the forearm and the CT remains smaller, i. e. the WFR remains little, these patients may suffer from otherwise (clinically) clear CTS without pathologic ratio of the CSAs of the median nerve in the forearm. Because of this effect, pathologic WFR used for nondiabetic patients will underestimate the likelihood of CTS in diabetic subjects.

Our study has several limitations. We have chosen a retrospective study design. As with all retrospective investigations, a preselection bias cannot be ruled out. However, the retrospective design enabled us to assess a rather large number of patients. Unfortunately our groups were heterogeneous. Even though there was no significant difference in age, we had more male patients in Group DM-CTS+. Due to the heterogeneity of our samples, we are unable to deduct reliably more information from our data than those regarding the WFR.

As a consequence to these findings, neither WFR nor CSA seems to be appropriate in diabetic patients for US confirmation of CTS. It has to be mentioned that prospective studies have to be carried out to confirm our findings. The extent of nerve enlargement in diabetic patients remains unknown. "Normal" CSA values for diabetic patients, including correlation to HbA1c levels, are needed. Studies on this topic would be highly desirable to elucidate this relevant field of nerve pathology.

$\begin{array}{ll}\text { ABBREVIATIONS } \\ \text { CSA } & \text { cross-sectional area } \\ \text { CT } & \text { carpal tunnel } \\ \text { CTS } & \text { carpal tunnel syndrome } \\ \text { DM } & \text { diabetes mellitus } \\ \text { HbA1c } & \text { hemoglobin A1c } \\ \text { NCS } & \text { nerve conduction studies } \\ \text { PNP } & \text { polyneuropathy } \\ \text { PQ } & \text { pronator quadratus muscle } \\ \text { US } & \text { ultrasound } \\ \text { WFR } & \text { wrist-to-forearm ratio }\end{array}$

\section{Conflict of Interest}

The authors declare that they have no conflict of interest.

\section{Reference}

[1] Latinovic R, Gulliford MC, Hughes RA. Incidence of common compressive neuropathies in primary care. Journal of neurology, neurosurgery, and psychiatry 2006; 77: 263-265

[2] Aboonq MS. Pathophysiology of carpal tunnel syndrome. Neurosciences 2015; 20: 4-9

[3] Wong SM, Griffith JF, Hui AC et al. Discriminatory sonographic criteria for the diagnosis of carpal tunnel syndrome. Arthritis and rheumatism 2002; 46: 1914-1921
[4] Atroshi I, Gummesson C, Johnsson R et al. Prevalence of carpal tunnel syndrome in a general population. Jama 1999; 282: $153-158$

[5] Mhoon JT, Juel VC, Hobson-Webb LD. Median nerve ultrasound as a screening tool in carpal tunnel syndrome: correlation of cross-sectional area measures with electrodiagnostic abnormality. Muscle \& nerve 2012; 46: $871-878$

[6] Ashraf AR, Jali R, Moghtaderi AR et al. The diagnostic value of ultrasonography in patients with electrophysiologicaly confirmed carpal tunnel syndrome. Electromyography and clinical neurophysiology 2009; 49: 3-8

[7] Visser LH, Smidt MH, Lee ML. High-resolution sonography versus EMG in the diagnosis of carpal tunnel syndrome. Journal of neurology, neurosurgery, and psychiatry 2008; 79: 63-67

[8] Moran L, Perez M, Esteban A et al. Sonographic measurement of crosssectional area of the median nerve in the diagnosis of carpal tunnel syndrome: correlation with nerve conduction studies. Journal of clinical ultrasound 2009; 37: 125-131

[9] Hammer HB, Hovden IA, Haavardsholm EA et al. Ultrasonography shows increased cross-sectional area of the median nerve in patients with arthritis and carpal tunnel syndrome. Rheumatology 2006; 45: 584-588

[10] Ziswiler HR, Reichenbach S, Vogelin E et al. Diagnostic value of sonography in patients with suspected carpal tunnel syndrome: a prospective study. Arthritis and rheumatism 2005; 52: 304-311

[11] Hobson-Webb LD, Massey JM, Juel VC et al. The ultrasonographic wristto-forearm median nerve area ratio in carpal tunnel syndrome. Clinical neurophysiology : official journal of the International Federation of Clinical Neurophysiology 2008; 119: 1353-1357

[12] Whiting DR, Guariguata L, Weil C et al. IDF diabetes atlas: global estimates of the prevalence of diabetes for 2011 and 2030. Diabetes research and clinical practice 2011; 94: 311 - 321

[13] Tesfaye S, Selvarajah D. Advances in the epidemiology, pathogenesis and management of diabetic peripheral neuropathy. Diabetes/metabolism research and reviews 2012; 28 (Suppl. 1): 8-14

[14] Sharma AK, Thomas PK, De Molina AF. Peripheral nerve fiber size in experimental diabetes. Diabetes 1977; 26: 689-692

[15] Hobson-Webb LD, Massey JM, Juel VC. Nerve ultrasound in diabetic polyneuropathy: correlation with clinical characteristics and electrodiagnostic testing. Muscle \& nerve 2013; 47: 379-384

[16] Watanabe T, Ito H, Morita A et al. Sonographic evaluation of the median nerve in diabetic patients: comparison with nerve conduction studies. Journal of ultrasound in medicine : official journal of the American Institute of Ultrasound in Medicine 2009; 28: 727 - 734

[17] Giannini C, Dyck PJ. Ultrastructural morphometric abnormalities of sura nerve endoneurial microvessels in diabetes mellitus. Ann Neurol 1994; 36: $408-415$

[18] Pitarokoili K, Kerasnoudis A, Behrendt V et al. Facing the diagnostic challenge: Nerve ultrasound in diabetic patients with neuropathic symptoms. Muscle Nerve 2016; 54: $18-24$

[19] Telleman JA, Grimm A, Goedee S et al. Nerve ultrasound in polyneuropathies. Muscle Nerve 2017. doi:10.1002/mus.26029

[20] Kwon BC, Jung KI, Baek GH. Comparison of sonography and electrodiagnostic testing in the diagnosis of carpal tunnel syndrome. The Journal of hand surgery 2008; 33: 65-71

[21] Hunderfund AN, Boon AJ, Mandrekar JN et al. Sonography in carpal tunnel syndrome. Muscle \& nerve 2011; 44: 485-491

[22] Koyuncuoglu HR, Kutluhan S, Yesildag A et al. The value of ultrasonographic measurement in carpal tunnel syndrome in patients with negative electrodiagnostic tests. European journal of radiology 2005; 56: 365 - 369 